\title{
Probing hadronic interactions with the Pierre Auger Observatory
}

\author{
Francesco Fenu ${ }^{a, b, *}$ on behalf of the Pierre Auger Collaboration \\ a INFN Torino, \\ Via Pietro Giuria 1, 10125, Torino, Italy \\ ${ }^{b}$ Dipartimento di Fisica, Università degli studi di Torino \\ Via Pietro Giuria 1, 10125, Torino, Italy \\ E-mail: spokespersons@auger.org
}

Full author list at: https: //www . auger.org/archive/authors_2021_09.html

\begin{abstract}
The study of ultra-high energy cosmic rays provides information about the hadronic interactions in an energy and kinematic region well beyond those explored in accelerators. The Pierre Auger Observatory is the largest cosmic ray observatory ever built $\left(3000 \mathrm{~km}^{2}\right)$, operating since 2004 in the Argentinian Pampa. Its hybrid design allows one to measure the longitudinal and lateral distribution of extensive air showers and the establishment of an almost model-independent, calorimetric, energy scale. The observatory is sensitive to the electromagnetic and to the muonic components of extensive air showers, which are directly related to the energy and type of the primary particle and to the hadronic interaction parameters. In this contribution an overview of the results obtained by the Auger collaboration in the field of particle physics will be given. We will show the proton-Air cross section at 38.7 and $55.5 \mathrm{TeV}$. Our data indicate that hadronic interaction models fail in the predictions of the muon densities on the surface, too low with respect to the measured ones. Possible future lines of research to investigate this inconsistency will be identified.
\end{abstract}

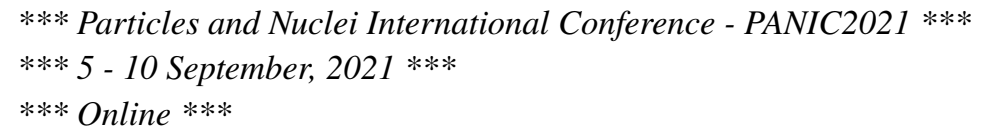

${ }^{*}$ Speaker 


\section{Introduction}

The Pierre Auger Observatory [1] is located in the Argentinian Pampa near the town of Malargue, at an altitude of $1400 \mathrm{~m}$. The Surface Detector (SD), covering an area of $3000 \mathrm{~km}^{2}$, consists of 1600 water-Cherenkov detectors deployed on a triangular grid of $1500 \mathrm{~m}$ side (the SD1500 array). A denser array of $24 \mathrm{~km}^{2}$, consisting of 64 detectors separated by $750 \mathrm{~m}$ (the SD750 array), is nested into the SD1500 array to lower the energy threshold. An array of 27 telescopes, the Fluorescence Detector (FD), overlooks the surface detectors allowing the measurement of the longitudinal profile of the cosmic ray showers through fluorescence emission. The energy of the primaries can be therefore determined in a calorimetric way and the $X_{\max }$ parameter, the slant depth of the shower maximum, can be measured. The purpose of this paper is to present the measurements of the Pierre Auger Observatory to investigate the hadronic interactions and discuss possible future developments.

\section{Studies on the $X_{\max }$ distribution}

The distribution of the $X_{\max }$ parameter bears information on the composition of the cosmic ray flux. The first two moments of the $X_{\max }$ distribution are for example used to determine the average mass and the purity of the cosmic ray flux. The full $X_{\max }$ distribution is also used to estimate the single primary fraction. As shown in [2], the composition is estimated to be light at around $10^{18.3}$ $\mathrm{eV}$ and to get progressively heavier toward the higher energies. Such studies are however affected by a non-negligible model dependence and some features of the data are not properly described by the models (see [2] and references therein).
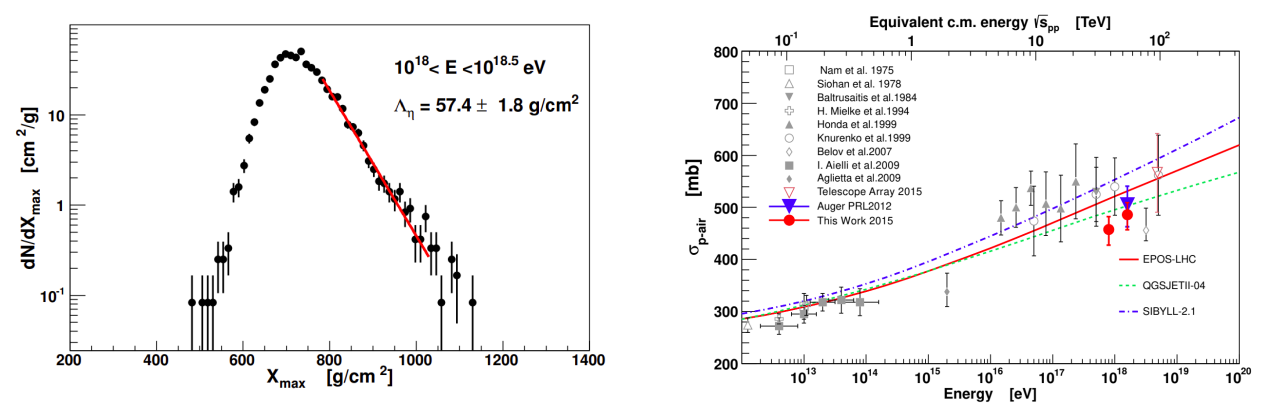

Figure 1: Left side: the $X_{\max }$ distribution with the exponential fit to determine $\Lambda_{\eta}$. Right side: the Pierre Auger estimation of the proton-air cross section in two bins of energy.

It has been shown that the steepness of the tail of the $X_{\max }$ distribution $\left(\Lambda_{\eta}\right)$ can be used to estimate the proton-air cross section. The hadronic cross section parameter is then rescaled in simulations till the simulated $\Lambda_{\eta}$ reproduces the one observed in data (see Fig. 1 left panel). The rescaling is performed according to:

$$
f\left(E, f_{19}\right)=1+\left(f_{19}-1\right) \frac{\log \left(E / 10^{15} \mathrm{eV}\right)}{\log \left(10^{19} \mathrm{eV} / 10^{15} \mathrm{eV}\right)}
$$

where $f_{19}$ is the rescaling parameter and $E$ is the energy of the secondary particle. This accounts for an energy dependent empirical correction that sets in at $10^{15} \mathrm{eV}$ and slowly increases with 
energy. The resulting cross section measured in two energy bins centered at $10^{17.9} \mathrm{eV}(38.7 \mathrm{TeV}$ c.m.s.) and $10^{18.25} \mathrm{eV}$ (55.5 TeV c.m.s.) [3] is shown in the right panel of Fig. 1. A moderate discrepancy with the extrapolation of the models to the highest energies is visible. The cross section is increasing with energy but the large uncertainty does not allow any further statements on the cross section evolution. The largest contribution to the systematic uncertainty comes from the possible contamination of the sample from a helium component. Assuming a 25\% Helium contamination, a $\sim 3 \%$ uncertainty on the cross section value has been estimated. Further smaller systematics are due to the uncertainty on the determination of the $\Lambda_{\eta}$ parameter and to the hadronic model uncertainty.

\section{The muon excess}

The design of the Pierre Auger Observatory does not, in general, allow the discrimination of muonic and electromagnetic component. To estimate the muonic component one has to operate in a regime where the electromagnetic component is not present anymore. The first possibility to perform such a measurement is to refer to events of the SD1500 array with a zenith angle greater than $60^{\circ}$ [4] (hereafter "inclined events"). In this condition the atmosphere absorbs the electromagnetic component and the residual signal is then to be interpreted as muonic signal alone. The signal size on ground, $N_{19}$, is obtained by fitting the muon distribution model to the detected signal ${ }^{1}$. We analyze hybrid events, events with a coincident measurement of both $N_{19}$ and the fluorescence signal. In this way it is possible to calculate $R_{\mu}$, an estimator of the muon content derived from $N_{19}$, as a function of the energy determined with the FD. In Fig. 2, left panel, it is shown the muon
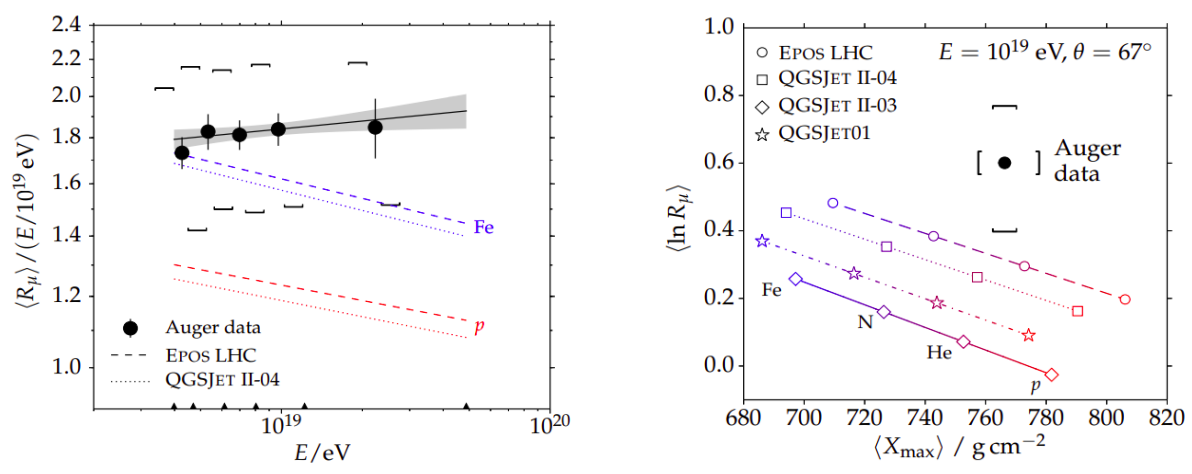

Figure 2: Left side: excess of the muon content $R_{\mu}$ divided by the energy, as a function of energy. Right side: correlation between the $X_{\max }$ and the muon content $R_{\mu}$

content (normalized to the energy) as a function of energy compared with the predictions from the models. As it is apparent, there is a strong deficit in the predicted muon size with respect to what observed in the data. On the right panel of Fig. 2, the correlation between the $X_{\max }$ parameter and the $R_{\mu}$ is shown. Data are represented as a point while predictions for different hadronic models and composition are represented as lines. As can be clearly seen, there is a strong disagreement between the predictions for the muon content and the $X_{\max }$. The $X_{\max }$ parameter, mostly driven by

\footnotetext{
${ }^{1}$ The model to describe the shape of the muon distribution is $\rho(\vec{r})=N_{19} \times \rho_{\mu, 19}(\vec{r}, \theta, \phi)$ where $\rho_{\mu, 19}$ is the expected signal from a $10^{19} \mathrm{eV}$ proton shower for a given direction and $N_{19}$ is the scaling parameter or the shower size.
} 
the electromagnetic component of the EAS, is well described while the predictions for the muonic component are clearly inadequate. A similar study has been also done with events detected by a set of 7 buried underground muon detectors [5] leading to the same conclusions as above.
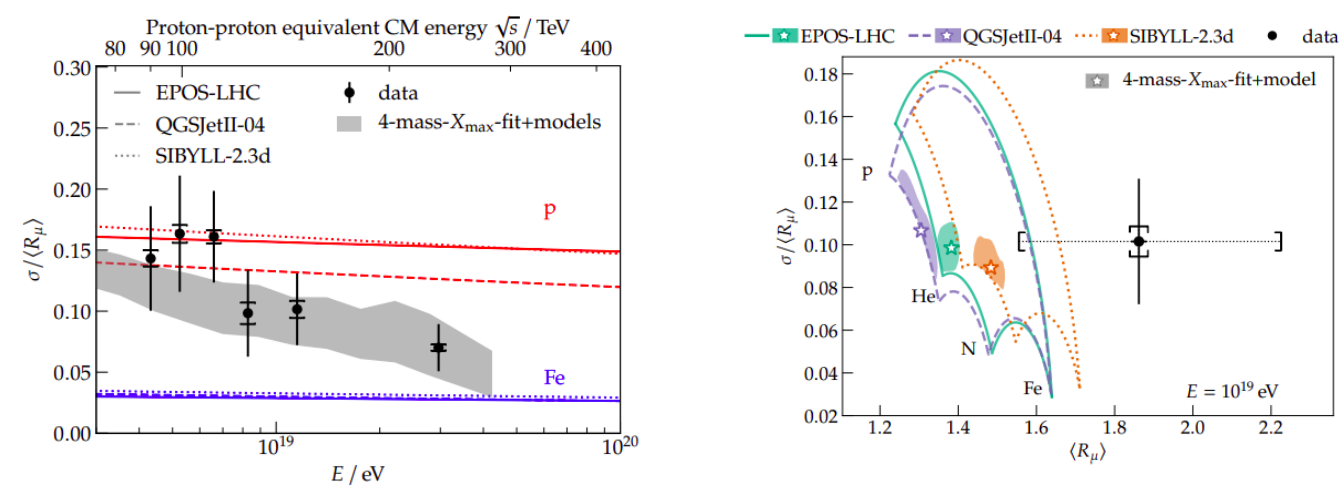

Figure 3: Left side: muon content fluctuation as a function of energy. Right side: correlation of the muon number fluctuation with the muon number.

An analysis based on inclined hybrid events allows one to measure the width of the muon content distribution, $R_{\mu}$ [6]. As shown in Fig. 3, left panel, the measured muon fluctuations are fully consistent with the predictions from the models. On the right panel we can instead see the correlation of the muon fluctuation with the muon content measurement. Again, it appears very clearly that, while the models fail in the description of the muon density, no discrepancy can be observed in the muon fluctuations. Further measurements of the muon excess have been performed with the SD detector [7] and studies on the nature of this excess have been presented in [8].

The muon issue has been detected with several other experiments [9] with an $8 \sigma$ global significance (obtained from a joint meta-analysis of all the experiments). The excess starts to emerge at around $\sim 10^{16} \mathrm{eV}$, which is also in the range of the LHC (in c.m.s. energy).

\section{Conclusions}

The strong muon excess undoubtedly observed in the data of several experiments is an indication of the inadequacy of the hadronic models at high energy. The picture is quite consistent as long as parameters like $X_{\max }$ are considered, but fails dramatically once parameters related to the hadronic component of the EAS are examined (see for example Fig. 2, right side). The correct predictions of the muon number fluctuations, together with the strong muon excess, show also that the inconsistency in the models cannot be confined to interactions at the very beginning of the shower. The statistical fluctuations on the first interactions would, under this hypothesis, be also amplified in a similar way as the muon excess itself. The fact that the fluctuations are correctly described points therefore to a small inconsistency of the models accumulating over several interactions.

To assess the sensitivity of the various EAS observables to changes in the hadronic interaction models, Monte Carlo based studies have been performed [10]. The parameters of the hadronic models have been rescaled in a similar way as in Eq. 1 to study their impact on the EAS development. The very high sensitivity of the total muonic content to changes in the $\pi^{0}$ fraction can be clearly 


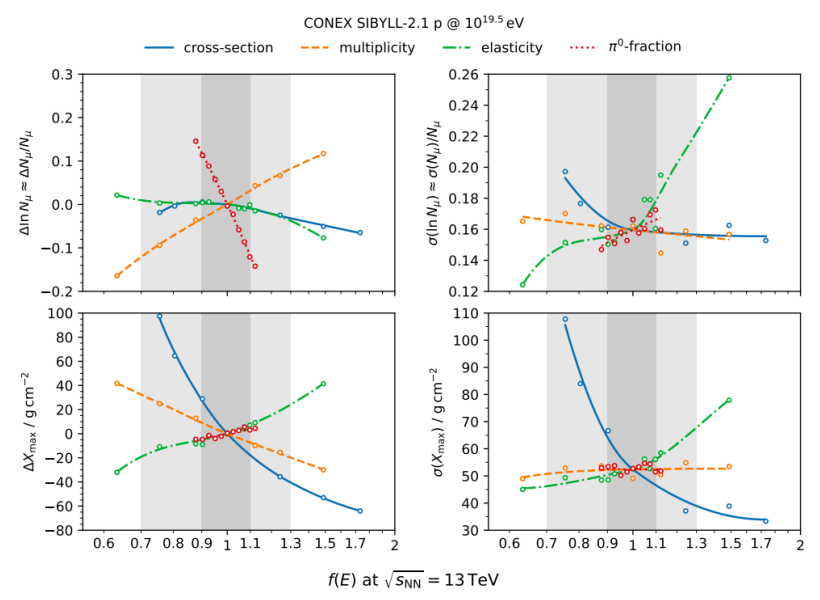

Figure 4: impact of the tuning of cross section, elasticity, multiplicity and $\pi^{0}$ fraction on the EAS observables. Top left: impact on the muonic number. Top right: impact on the muonic fluctuations. Bottom left: impact on the $X_{\max }$. Bottom right: impact on the $X_{\max }$ fluctuations.

seen in Fig. 4. The same change on the $\pi^{0}$ fraction does not seem to heavily affect either the muon fluctuations, or the $X_{\max }$ or the $X_{\max }$ fluctuations. Models that predict a lower $\pi^{0}$ fraction are therefore favored. All the other parameters like the multiplicity, cross section or elasticity are less likely to explain the observed scenario.

A great limitation of the current hadronic models (besides the large extrapolation in energy) is the fact that they have been tuned on data from proton-proton collisions and on low pseudorapidity accelerator data. Models are therefore not satisfactory in the description of the muon predictions for highly forward regions and nucleon-air collisions. The forthcoming Run 3 at the LHC, for which proton-oxygen and oxygen-oxygen collisions have been approved, can therefore bring useful data to solve this puzzle. Another fundamental line of action is represented by the upgrade of the Pierre Auger Observatory, AugerPrime [11], currently underway, whose main goal will be that of improving the sensitivity to the composition at all the energies.

\section{References}

[1] A. Aab et al., Nucl. Instrum. Meth. A 798, 172 (2015)

[2] A. Yushkov, PoS(ICRC2019)482 (2020) - (And references therein)

[3] R. Ulrich, PoS(ICRC2015)401 (2015) - (And references therein)

[4] A. Aab et al., Phys. Rev. D 91, 032003 (2015)

[5] A. Aab et al., Eur. Phys. J. C 80:751 (2020)

[6] A. Aab et al., Phys. Rev. Lett. 126, 152002 (2021)

[7] A. Aab et al., Phys. Rev. D 96, 122003 (2021)

[8] A. Aab et al., Phys. Rev. Lett. 117, 192001 (2016)

[9] D. Soldin, PoS(ICRC2021)349 (2021)

[10] R. Ulrich et al., Phys. Rev. D 83, 054026 (2011)

[11] A. Aab et al., arXiv:1604.03637 (2016) 